\title{
Structural Variations of Metallothionein with or without Zinc Ions \\ Elucidated by All-Atom Molecular Dynamics Simulations
}

\author{
AUTHOR NAMES \\ Rikuri Morita*, Yasuteru Shigeta, Ryuhei Harada*
}

\section{AUTHOR ADDRESSES}

Center for Computational Sciences, University of Tsukuba, 1-1-1 Tennohdai, Tsukuba, Ibaraki 305-8577, Japan 


\section{Figure S1}

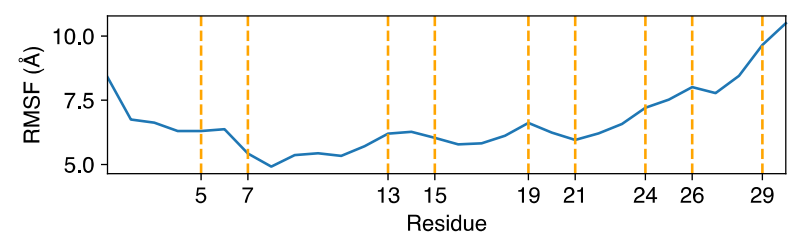

Root mean square fluctuation of the $\mathrm{C} \alpha$ atom of the $\beta$-domain of Holo-MT. The positions of the nine cysteines are indicated by dashed lines. 
Figure S2
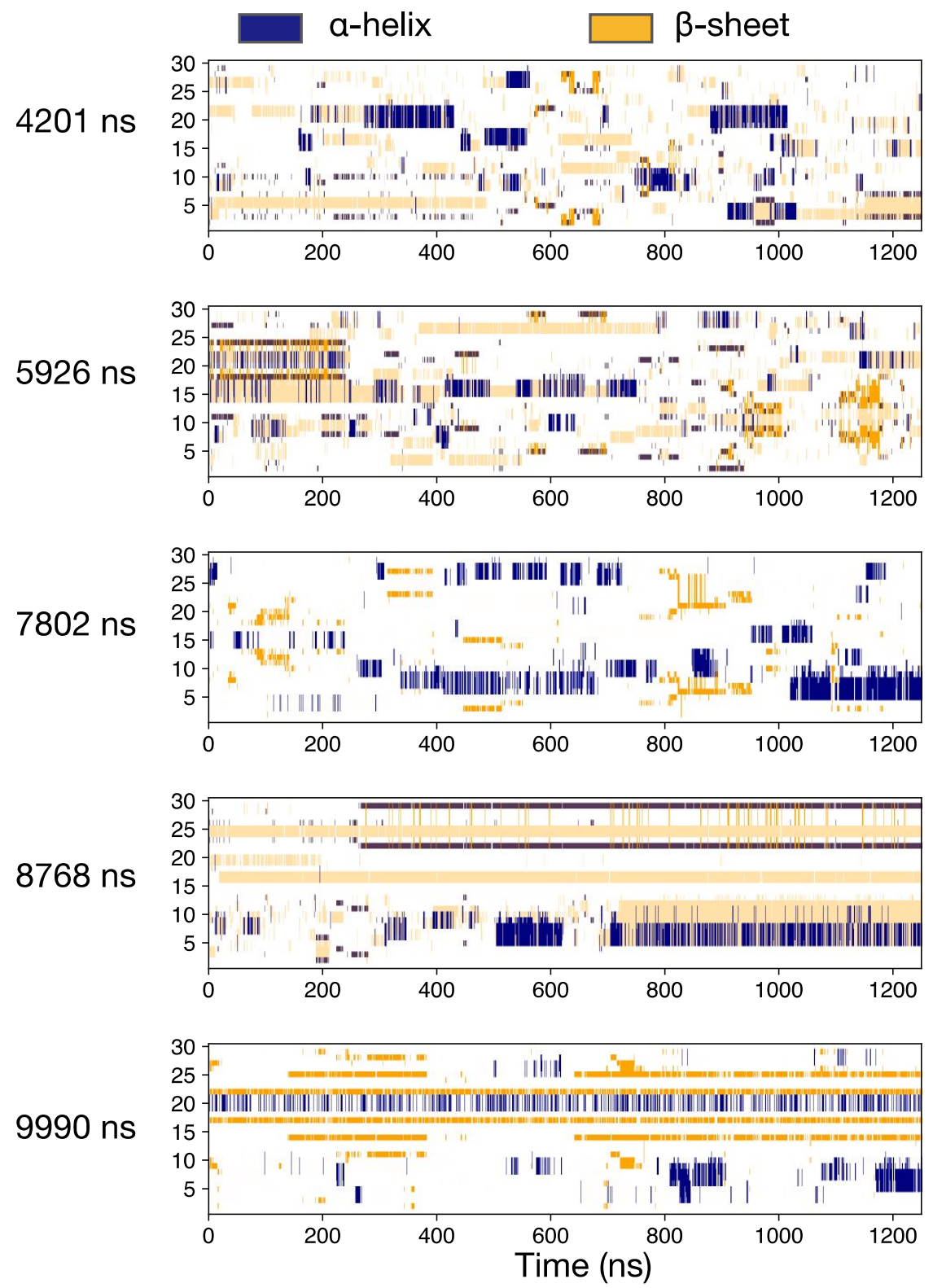

Time series of secondary structures for the MD simulation of the Apo-MT structure solvated into the Holo-MT condition. In each time series, $\alpha$-helix (navy) and $\beta$-sheet (orange, light orange) are shown. 
Figure S3

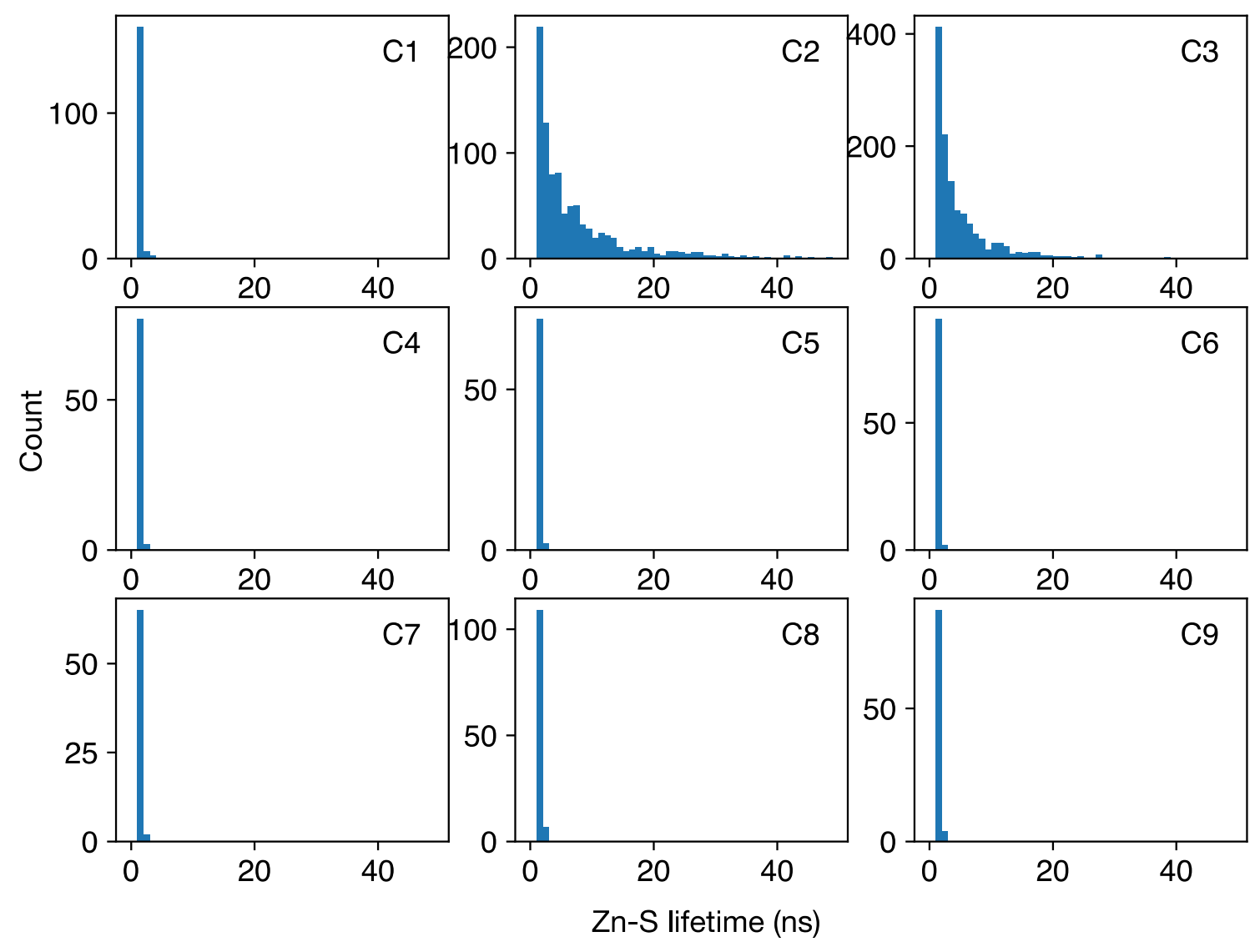

Distribution of the lifetime of the $\mathrm{Zn}^{2+}-\mathrm{S}$ coordinate bond. The lifetime was kept counting when the $\mathrm{Zn}^{2+}$-S distance was less than $4 \AA$. The time step used for the lifetime calculation was $1 \mathrm{~ns}$. 
Figure S4
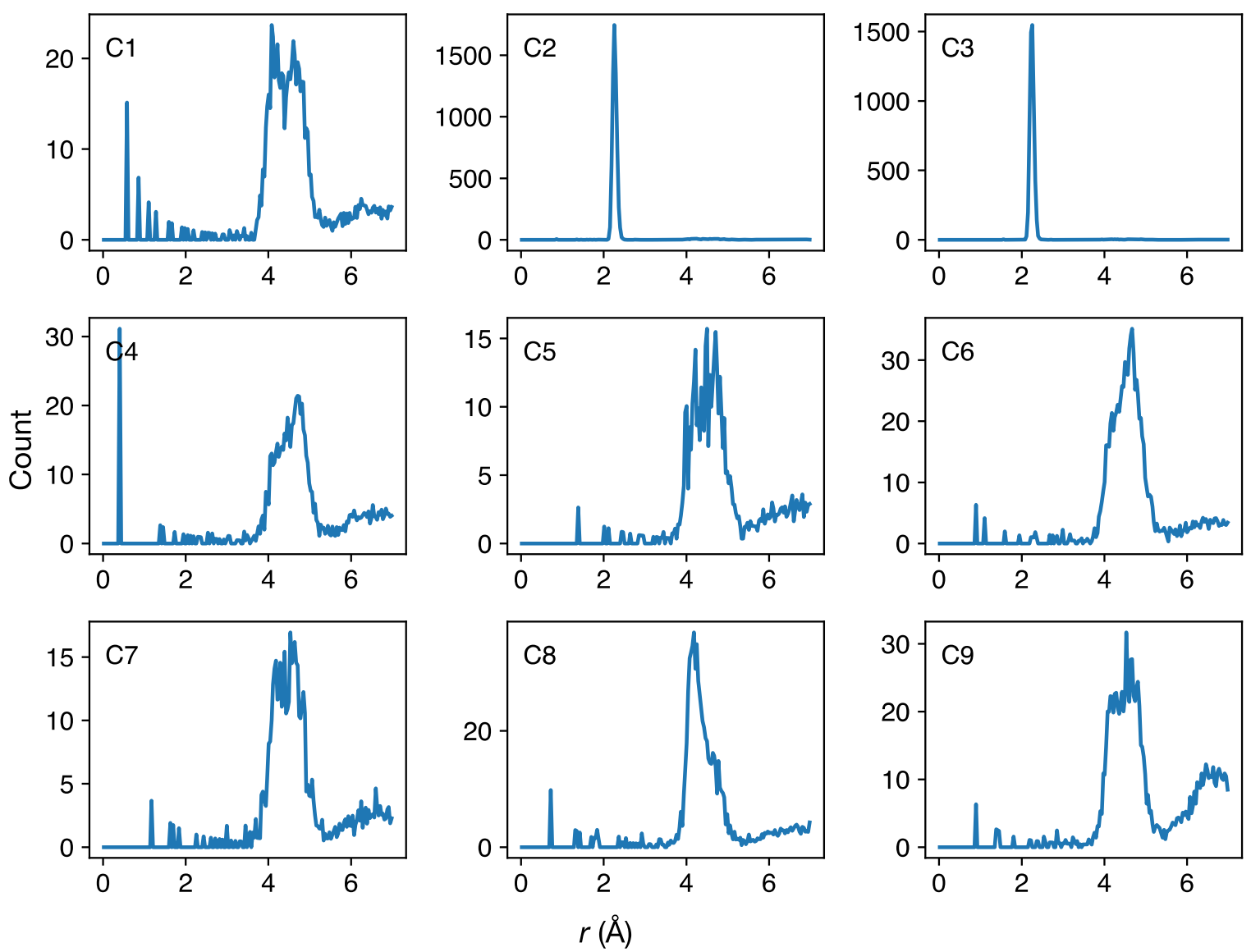

Radial distributions of the $\mathrm{Zn}^{2+}$ ions around each sulfur atom of C1-C9. 
Figure S5
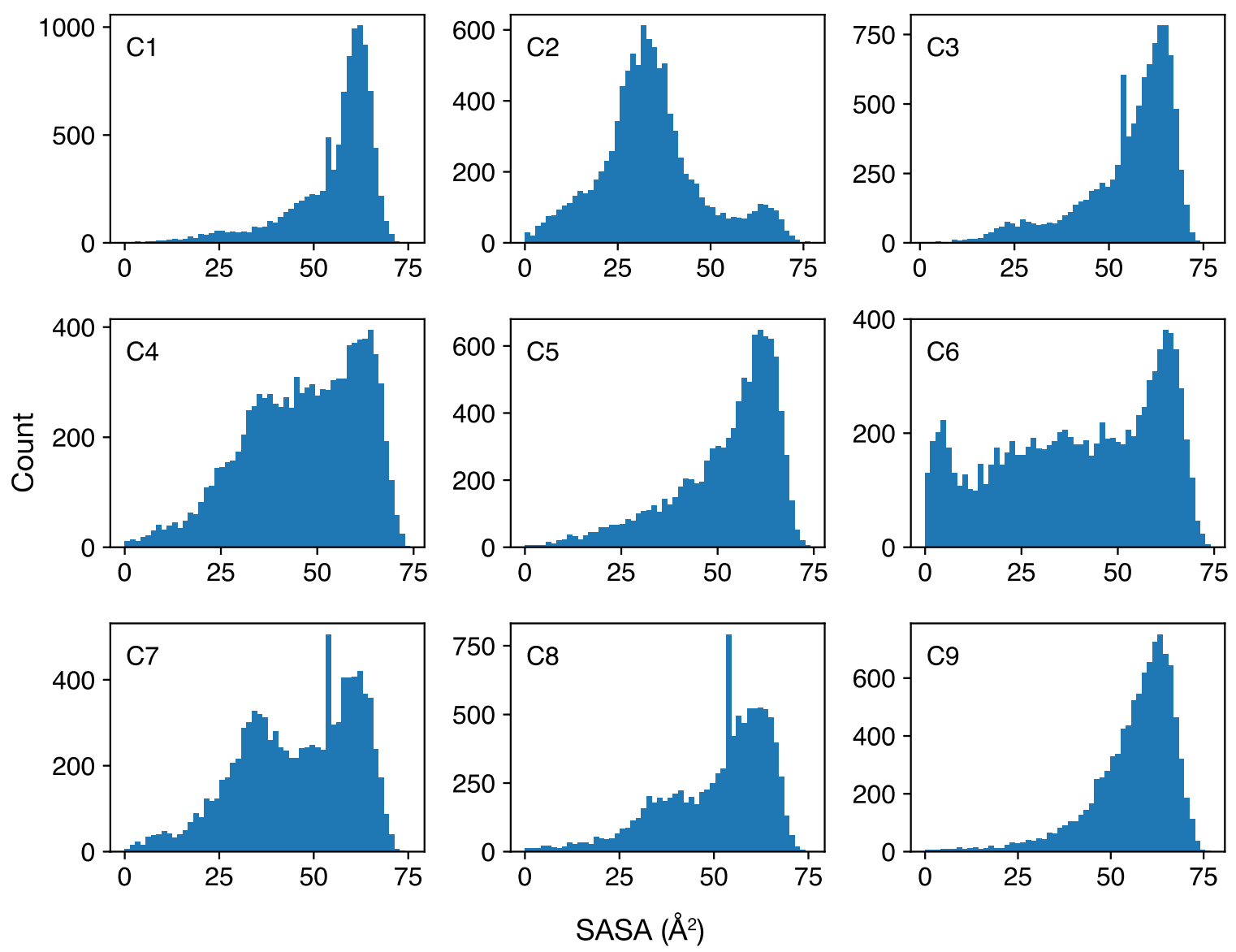

Distributions of each solvent accessible surface area (SASA) of each sulfur atom of C1-C9. 